\title{
Face Recognition Using a Gabor Filter Bank Approach
}

\author{
Walid R. Boukabou \\ School of Computer Science \\ Queen's University Belfast \\ Belfast BT7 1NN, UK \\ wboukabou01@qub.ac.uk
}

\author{
Lahouari Ghouti \\ School of Computer Science \\ Queen's University Belfast \\ Belfast BT7 1NN, UK \\ l.ghouti@qub.ac.uk
}

\author{
Ahmed Bouridane \\ School of Computer Science \\ Queen's University Belfast \\ Belfast BT7 1NN, UK \\ a.bouridane@qub.ac.uk
}

\begin{abstract}
Face recognition is a challenging field of research not only because of the complexity of this subject, but also because of its numerous practical applications. Much progress has been made towards recognising faces under controlled conditions, especially under normalised pose and lighting conditions and with neutral expression. However, the recognition of face images acquired in an outdoor environment with changes in illumination and/or pose remains a largely unsolved problem. This is due to the fact that most of face recognition methods assume that the pose of the face is known.

In this paper, we propose the use of a Gabor Filter Bank to extract an augmented Gabor-face vector to solve the pose estimation problem, extract some statistical features such as means and variances. And then the classification is performed using the nearest neighbour algorithm with the Euclidean distance. Finally, experimental results are reported to show the robustness of the extracted feature vectors for the recognition problem.
\end{abstract}

\section{Introduction}

During the last few years, face recognition has received a lot of attention from researchers from many areas such as pattern recognition, computer vision, biometrics, neural networks and image processing. This common interest is not only motivated by the complexity of this subject, but also by its numerous practical applications such as bank card identification, access control, mug shots searching, security monitoring and surveillance systems.

Much progress has been made towards recognising faces under controlled conditions [6],[11](especially for faces under normalised pose and lighting conditions and with neutral expression). However, recognition of face images acquired in an outdoor environment with changes in illumination and/or pose remains a largely unsolved problem. In other words, current systems are still far away from the capability of the human perception system.

This paper introduces a Gabor Wavelet Classification method for face recognition. The processing of facial images by a Gabor filter is chosen for its biological relevance and technical properties. The Gabor filter kernels have similar shapes as the receptive fields of simple cells in the primary visual cortex [8]. They are multi-scale and multi-orientation kernels. The Gabor transformed face images yield features that display scale, locality, and differentiation properties. These properties are quite robust to variability of face image formation, such as the variations of illumination, head rotation and facial expressions.

The rest of this paper is organized as follows. A brief review of facial feature extraction using Gabor Wavelet functions is described in section 2. In section 3 , the classification method by the nearest neighbour algorithm is presented. The experimental results are shown in Section 4. And finally some conclusions are given in Section 5.

\section{Face Feature Extraction}

\subsection{Gabor Functions and Wavelets}

The two-dimensional Gabor Wavelets function $g(x, y)$ and its Fourier transform $G(u, v)$ can be defined as follows[4]

$$
\begin{aligned}
& g(x, y)=\frac{1}{2 \pi \sigma_{x} \sigma_{y}} \exp \left[-\frac{1}{2}\left(\frac{x^{2}}{\sigma_{x}{ }^{2}}+\frac{y^{2}}{\sigma_{y}{ }^{2}}\right)+2 \pi j W x\right] \\
& G(u, v)=\exp \left[-\frac{1}{2}\left(\frac{(u-W)^{2}}{\sigma_{u}{ }^{2}}+\frac{v^{2}}{\sigma_{v}{ }^{2}}\right)\right]
\end{aligned}
$$

Where $\sigma_{u}=1 / 2 \pi \sigma_{x}$ and $\sigma_{v}=1 / 2 \pi \sigma_{y}$. Gabor functions can form a complete but nonorhgonal basis set. Expanding a signal using this basis provides a 
localized frequency description. A class of self-similar functions, referred to as Gabor wavelets in the following discussion, is now considered. Let $g(x, y)$ be the mother Gabor wavelet, then this self-similar filter dictionary can be obtained by appropriate dilations and rotations of $\mathrm{g}(\mathrm{x}, \mathrm{y})$ through the generating function:

$$
\begin{aligned}
& g_{m n}(x, y)=a^{-m} G\left(x^{\prime}, y^{\prime}\right) \\
& x^{\prime}=a^{-m}(x \cos \theta+y \sin \theta) \\
& x^{\prime}=a^{-m}(-x \sin \theta+y \cos \theta)
\end{aligned}
$$

where $a>1, \mathrm{~m}, \mathrm{n}=$ integer and $\theta=n \pi / k$ is the orientation ( $\mathrm{k}$ is the number of orientations) and $\mathrm{a}^{-\mathrm{m}}$ is the scale factor.

\subsection{Gabor Filter Dictionary Design}

The nonorthogonality of the Gabor wavelets implies that there is redundant information in the filtered images, and the following strategy is used to reduce this redundancy. Let $U_{l}$ and $U_{h}$ denote the lower and upper centre frequencies of interest. Let $K$ be the number of orientations and $\mathrm{S}$ be the number of scales in the multiresolution decomposition. Then the design strategy is to ensure that the half-peak magnitude support of the filter responses in the frequency spectrum touch each other [4]. This results in the following formulas for computing the filter parameters $\sigma_{\mathrm{u}}$ and $\sigma_{\mathrm{v}}$ (and thus $\sigma_{\mathrm{x}}$ and $\sigma_{\mathrm{y}}$ ).

$$
\begin{gathered}
\mathrm{a}=\left(\mathrm{U}_{\mathrm{h}} / \mathrm{U}_{\mathrm{l}}\right)^{-\frac{1}{\mathrm{~s}-1}} \\
\sigma_{u}=\frac{(a-1) U_{h}}{(a+1) \sqrt{2 \ln 2}} \\
\sigma_{v}=\tan \left(\frac{\pi}{2 k}\right)\left[U_{h}-2 \ln \left(\frac{\sigma_{u}^{2}}{U_{h}}\right)\right]\left[2 \ln 2-\frac{(2 \ln 2)^{2} \sigma_{u}^{2}}{U_{h}^{2}}\right]^{\frac{-1}{2}}
\end{gathered}
$$

Where $\mathrm{W}=U_{h}$, and $m=0,1, \ldots, S$ - 1 . In order to eliminate the sensitivity of the filter response to absolute intensity values, the real (even) components of the 2D Gabor filters are biased by adding a constant to make them zero mean.

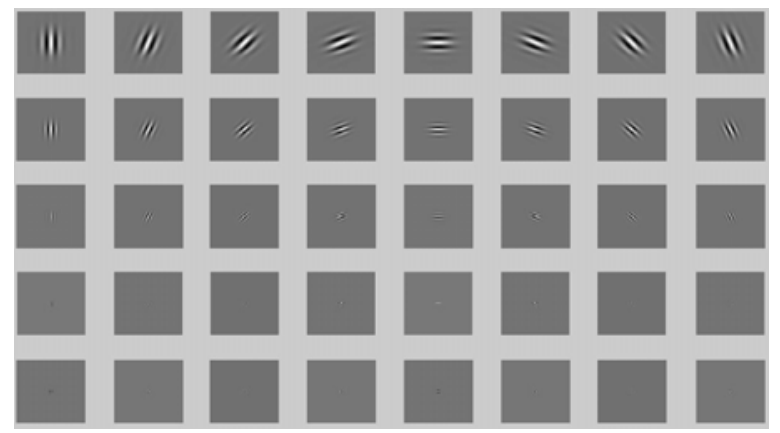

Figure 1. Real part of Gabor Filter Bank. The filter parameters used are $K=8, S=5$, $\mathrm{U}_{\mathrm{l}}=\mathbf{0 . 0 5}, \mathrm{U}_{\mathrm{h}}=\mathbf{0 . 4}$

\subsection{Features Extraction}

Given any image $I(x, y)$, its Gabor wavelet transformation is then defined to be:

$W_{m n}=\int I\left(x_{1}, y_{1}\right) g_{m n}^{*}\left(x-x_{1}, y-y_{1}\right) d x_{1} d y_{1}$

where * indicates the complex conjugate. The Gabor wavelet transformation of the facial image is calculated at five scales, $m \in\{0,1,2,3,4\}$ and eight different orientations $n \in\{0, \ldots, 7\}$, and let us set (as shown in Figure 1) $\mathrm{U}_{\mathrm{l}}=0.05$ and $\mathrm{U}_{\mathrm{h}}=0.4$.

$W_{m n}$ denotes a Gabor wavelet transformation of a face. The augmented Gabor-face vector can be defined then as follows [2]:

$$
\chi=\left(W_{0,0}{ }^{t}, \ldots \ldots, W_{4,7}{ }^{t}\right)^{t}
$$

where $t$ is the transpose operator. The augmented Gabor-face vector can encompass all facial Gabor wavelet transformations, and has important discriminatory information such as robustness against varying expression and pose in the face image.

Figure 2 shows the Gabor Filter Bank applied to a single face image from Yale Database [10]

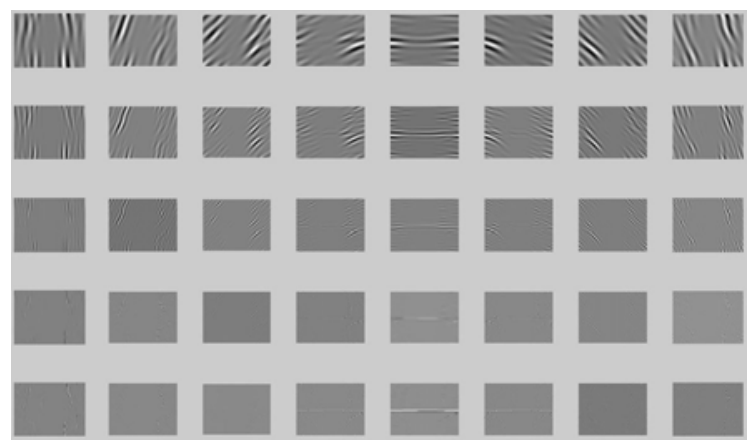

Figure 2. Real part of a face image transformed using the Gabor Filter Bank.

A feature vector can be now constructed. For each element $W_{m n}$, from the augmented Gabor-face vector, statistical features of the image are computed such as the mean and the variance (or standard deviation) $\mu_{m n}$ and $\sigma_{m n}$. In the experiments, we show that the best results are obtained if five scales $(\mathrm{S}=5)$ and eight orientations $(K=8)$ are used. The resulting feature vector is as follows:

$f=\left[\mu_{0,0}, \sigma_{0,0}, \mu_{0,1}, \sigma_{0,1}, \ldots, \mu_{4,7}, \sigma_{4,7}\right]$

For each face image, we will obtain an 80 element feature vector.

\section{Distance Measure}

The problem of finding the closest point in highdimensional spaces is common in pattern recognition problems. Unfortunately, the complexity of most 
existing search algorithms grows exponentially with the dimension, making them impractical for dimensionality above 15 [3],[5]. However, the nearest neighbour classifier (NNC) remains one of the simplest and most efficient algorithms in pattern recognition. It approximates the minimum error Bayesian classifier in the limit as the number of reference vectors gets large (large database).

In this work, we have used a nearest neighbour classifier with the Euclidean distance: If we consider two face images $i$ and $j$, let $\mathrm{f}^{(\mathrm{i})}$ and $\mathrm{f}^{(\mathrm{j})}$ represent the corresponding feature vectors. Then the distance $d_{i j}$ between the two patterns in the feature space is defined to be:

$$
d_{i j}=\sqrt{\sum_{n} \sum_{m}\left[\left(\frac{\mu_{m n}^{(i)}-\mu_{m n}^{(j)}}{\alpha\left(\mu_{m n}\right)}\right)^{2}+\left(\frac{\sigma_{m n}^{(i)}-\sigma_{m n}^{(j)}}{\alpha\left(\sigma_{m n}\right)}\right)^{2}\right]}
$$

$\alpha\left(\mu_{m n}\right)$ and $\alpha\left(\sigma_{m n}\right)$ are the standard deviations of the respective features over the entire database, and are used to normalize the individual feature components.

\section{Experimental Results}

In order to test the efficiency of the method presented above, we performed series of experiments using the Yale Face Database. This is a collection of 165 images of 15 individuals. The images that belong to the same person (same class) present variations in expression and illumination. Sample images from the database are displayed in figures 3 .
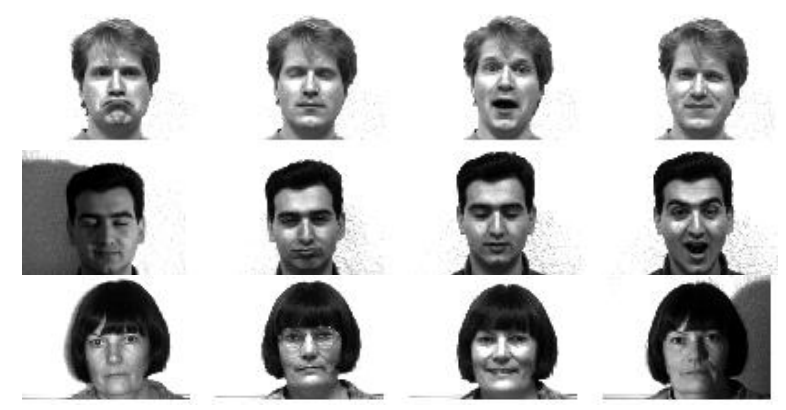

Figure 3. Sample images from Yale Face Database with different expressions and illumination conditions

The following experiments were performed using the images of the Yale database. Since 11 images of each individual are available (different expressions: happy, sad, sleepy... and different lighting sources: centre, left and right), we use some of them as reference faces (to compare with them) and the others as input data (test images).

\subsection{Experiment 1}

In order to define the ideal parameters for our Gabor Filter Bank, we changed some the parameters and calculated the successful rates using a reduced database: we used in this experiment 30 face images as reference faces and 30 other images as test faces. The parameters which have been changed are $S$ and $K$ (the number of scales and the number of orientations). The results obtained are displayed in table 1

Table1. Results for experiment 1

\begin{tabular}{|c|c|c|}
\hline $\begin{array}{c}\text { GFB } \\
\text { parameters }\end{array}$ & $\begin{array}{c}\text { Number of } \\
\text { Misclassification } \\
\text { s }\end{array}$ & $\begin{array}{c}\text { Recognition } \\
\text { Rate }\end{array}$ \\
\hline $\begin{array}{c}\mathrm{S}=3 \\
\mathrm{~K}=4\end{array}$ & 7 & $76.67 \%$ \\
\hline $\begin{array}{c}\mathrm{S}=4 \\
\mathrm{~K}=4\end{array}$ & 6 & $80.00 \%$ \\
\hline $\begin{array}{c}\mathrm{S}=4 \\
\mathrm{~K}=6\end{array}$ & 4 & $86.67 \%$ \\
\hline $\begin{array}{c}\mathrm{S}=5 \\
\mathrm{~K}=8\end{array}$ & 2 & $93.33 \%$ \\
\hline $\mathrm{S}=6$ & 2 & $93.33 \%$ \\
$\mathrm{~K}=9$ & & \\
\hline
\end{tabular}

It can be seen from these results that the recognition rate for the reduced dataset increases significantly from $77 \%$ to $93 \%$, which means that the higher the order of the GFB is, the better the results are. However, if they exceed 5 scales and 8 orientations, the recognition rate does not change a lot. This is due to the fact that at the sixth scale, we don't get much more new information.

Although, it is important to mention that the time of execution grows remarkably when we augment the order of the GFB.

\subsection{Experiment 2}

We aim in this experiment to show how the database size affects the recognition rate. To do so, we took randomly a defined number of images for each person (class), put the images all together in a dataset, and then calculate the distances from each image to all the others. Finally we compared the distances with a view to assess if the minimum distance corresponds to the same class (person). In this experiment we took 
$\mathrm{K}=8$ and $\mathrm{S}=5$ as parameters for the Gabor Filter Bank. The results obtained are illustrated in Table 2.

Table2. Successful rates over database size

\begin{tabular}{|c|c|c|}
\hline $\begin{array}{c}\text { Number of } \\
\text { Images in } \\
\text { the } \\
\text { Database }\end{array}$ & $\begin{array}{c}\text { Number of } \\
\text { Misclassification } \\
\text { s }\end{array}$ & $\begin{array}{c}\text { Recognition } \\
\text { Rate }\end{array}$ \\
\hline 30 & 2 & $93.33 \%$ \\
\hline 60 & 5 & $91.67 \%$ \\
\hline 90 & 11 & $87.78 \%$ \\
\hline 150 & 21 & $86.00 \%$ \\
\hline
\end{tabular}

From the above table, it is clear that the recognition rate decreases when the database size increases. However, it can bee seen that an increase of the size of the database by a factor of 5 only results in an increase of $7 \%$ of the recognition rate.

\section{Conclusion}

In this paper we have proposed a method for face recognition based on Gabor Filter Banks. We have shown that a Gabor filter yields robustness against expression, illumination and small rotations. This has been demonstrated by a recognition rate of $93 \%$ even by using very simple statistical features such as means and variances and a basic metric such as Euclidean distance.

As an extension of this work, we believe that it would be interesting to extract more discriminant statistical features from the filtered images. That will not increase much the size of the feature vector but it will affect the time of executing.

\section{References}

[1] P. Belhumeur, J. Hespanha, and D. Kriegman, "Eigenfaces vs. Fisherfaces: Recognition using class specific linear projection," IEEE Trans. on Pattern Analysis and Machine Intelligence, vol. 19, no. 7, 1997.

[2] G. Dai, and C. Zhou, "Face Recognition Using Support Vector Machines with the Robust Feature," Proc. of the 2003 IEEE International Workshop on Robot and Human interactive Communication Millbrae, California, USA. Oct. 31 -Nov. 2, 2003.

[3] Anil K. Jain, Robert P.W. Duin, and Jianchang Mao, "Statistical Pattern Recognition: A Review," IEEE Trans. on Pattern Analysis and Machine Intelligence, vol. 22, no. 1, 2000.
[4] B.S. Manjunath, and W.Y. Ma, "Texture Features for Browsing and Retrieval of Image Data," IEEE Trans. on Pattern Analysis and Machine Intelligence, vol. 18, NO. 8, Aug. 1996.

[5] Sameer A. Nene, and Shree K. Nayar, "A Simple Algorithm for Nearest Neighbor Search in High Dimensions," IEEE Trans. on Pattern Analysis and Machine Intelligence, vol. 19, no. 9, 1997.

[6] P. J. Phillips, P. J. Flynn, T. Scruggs, K. W. Bowyer, J. Chang, K. Hoffman, J. Marques, J. Min, and W. Worek, "Overview of the Face Recognition Grand Challenge," Proc. of the IEEE Computer Society Conference on Computer Vision and Pattern Recognition, 2005.

[7] M. Turk, and A. Pentland, "Face recognition using eigenfaces," In Proc. IEEE Conference on Computer Vision and Pattern Recognition, 1991.

[8] L. Wiskott, J.M. Fellous, N. Krger, and C. von der Malsburg, "Face Recognition by Elastic Bunch Graph Matching," IEEE Trans. on Pattern Analysis and Machine Intelligence, vol. 19, no. 7, pp. 775-779, 1997.

[9] J. D. Woodward Jr., C. Horn, J. Gatune, and A. Thomas, "Biometrics A Look at Facial Recognition," RAND Public Safety and Justice for the Virginia State Crime Commission, 2003.

[10] The Yale Face Database.

[11] W. Zhao, R. Chellappa, A. Rosenfeld, and P.J. Phillips, "Face recognition: A literature survey," ACM Computing Surveys, Vol. 35, No. 4, Dec. 2003. 